\title{
Quantitative Analysis of Antiretroviral Drugs in Lysates of Peripheral Blood Mononuclear Cells Using MALDI-Triple Quadrupole Mass Spectrometry
}

\author{
Jeroen J. A. van Kampen, ${ }^{\dagger, \neq}$ Peter C. Burgers, ${ }^{\dagger}$ Rob A. Gruters, ${ }^{\S}$ Albert D. M. E Osterhaus, ${ }^{\S}$ \\ Ronald de Groot," Theo M. Luider, ${ }^{\dagger}$ and Dietrich A. Volmer*, ${ }^{*}$ \\ Department of Neurology, Laboratory of Neuro-Oncology and Clinical \& Cancer Proteomics, Erasmus MC, \\ Rotterdam, The Netherlands, Department of Paediatrics, Division of Paediatric Infectious Diseases \& Immunology, \\ Erasmus MC-Sophia, The Netherlands, Department of Virology, Erasmus MC, Rotterdam, The Netherlands, \\ Department of Paediatrics, UMC St. Radboud, Nijmegen, The Netherlands, and Medical Research Council, Human \\ Nutrition Research, Cambridge, United Kingdom
}

We report here on the use of a prototype matrix-assisted laser desorption/ionization (MALDI)-triple quadrupole mass spectrometer for quantitative analysis of six antiretroviral drugs in lysates of peripheral blood mononuclear cells (PBMC). Of the five investigated MALDI matrixes, 2,5-dihydroxybenzoic acid (DHB) and the novel 7-hydroxy-4-(trifluoromethyl)coumarin (HFMC) showed the broadest application ranges for the antiretroviral drugs. For DHB, the mean relative errors ranged from 8.3 (ritonavir) to $4.3 \%$ (saquinavir). The mean precisions (CV) ranged from 17.3 (nevirapine) to $10.8 \%$ (saquinavir). The obtained lower limits of quantitation (LLOQ) readily allow clinical applications using just 1 million PBMC from HIV-infected patients under therapy. The new matrix HFMC was used for quantitative analysis of the HIV protease inhibitor indinavir using a stainless steel target plate as well as a target plate with a novel, strongly hydrophobic fluoropolymer coating. Using the coated target plate, the mean relative error improved from 10.1 to $4.6 \%$, the mean precision from 33.9 to $9.9 \% \mathrm{CV}$, and the LLOQ from 16 to $1 \mathrm{fmol}$. In addition, the measurement time for one spot went down from 6 to only $2.5 \mathrm{~s}$.

Matrix-assisted laser desorption/ionization (MALDI) and electrospray ionization (ESI) are extensively used as ionization techniques for mass spectrometric analysis of biological samples. For qualitative and quantitative analysis of compounds such as pharmaceutical drugs, with molecular masses below $1000 \mathrm{Da}$, ESI mass spectrometry is usually preferred over MALDI, because analysis of small molecules with MALDI is often hampered by matrix-derived chemical noise in the low $m / z$ range. MALDI,

\footnotetext{
* To whom correspondence should be addressed. Tel. +44 1223437550 . Fax +44 122343 7515. E-mail: Dietrich.Volmer@mrc-hnr.cam.ac.uk.

${ }^{\dagger}$ Department of Neurology, Laboratory of Neuro-Oncology and Clinical \& Cancer Proteomics, Erasmus MC.

* Department of Paediatrics, Division of Paediatric Infectious Diseases \& Immunology, Erasmus MC-Sophia.

$\S$ Department of Virology, Erasmus MC.

"UMC St. Radboud.

${ }^{\perp}$ Medical Research Council.
}

however, does offer important advantages over ESI, namely, the much higher sample throughput and its insensitivity to ion suppression. ${ }^{1,2}$ In addition, samples can be conveniently stored on the target plates for future (re)analysis. ${ }^{3}$

Various approaches have been described to overcome the problem of matrix-derived chemical noise, including carefully optimized matrix-to-analyte ratios, ${ }^{4}$ the use of specialized additives, ${ }^{5}$ the use of ionic liquid matrixes ${ }^{6}$ or inorganic matrixes, ${ }^{7,8}$ or the application of high molecular weight matrixes. ${ }^{9-13}$ The interfering noise can also conveniently be circumvented by using tandem mass spectrometry (MS/MS). In the selected reaction monitoring mode (SRM) of a triple quadrupole mass spectrometer, interfering isobaric matrix ions are removed by monitoring only compound-specific precursor/product ion transitions. ${ }^{2}$

Quantitative analysis of small molecules using MALDI mass spectrometry is further complicated by poor reproducibilities of ion signals from heterogeneous crystal layers. The precisions can be improved, however, by using optimized cocrystallization techniques for sample and matrix, for example, with prestructured

(1) Volmer, D. A.; Sleno, L.; Bateman, K.; Sturino, C.; Oballa, R.; Mauriala, T.; Corr, J. Anal. Chem. 2007, 79, 9000-9006.

(2) Yang, Y.; Zhang, S.; Howe, K.; Wilson, D. B.; Moser, F.; Irwin, D.; Thannhauser, T. W. L. Biomol. Techmol. 2007, 18, 226-237.

(3) Dekker, L. J.; Burgers, P. C.; Guzel, C.; Luider, T. M. L. Chromatogr., B: Anal. Technol. Biomed. Life Sci. 2007, 847, 62-64.

(4) McCombie, G.; Knochenmuss, R. Anal. Chem. 2004, 76, 4990-4997.

(5) Guo, Z.; Zhang, Q.; Zou, H.; Guo, B.; Ni, J. Anal. Chem. 2002, 74, 16371641.

(6) Tholey, A.; Heinzle, E. Anal. Bioanal. Chem. 2006, 386, 24-37.

(7) Wei, J.; Buriak, J. M.; Siuzdak, G. Nature 1999, 399, 243-246.

(8) Kang, M. J.; Pyun, J. C.; Lee, J. C.; Choi, Y. J.; Park, J. H.; Park, J. G.; Lee, J. G.; Choi, H. J. Rapid Commun. Mass Spectrom. 2005, 19, 3166-3170.

(9) Ayorinde, F. O.; Eribo, B. E.; Johnson, J. H., Jr.; Elhilo, E. Rapid Commun. Mass Spectrom. 1999, 13, 1124-1128.

(10) Chen, Y. T.; Ling, Y. C. I. Mass Spectrom. 2002, 37, 716-730.

(11) Ling, Y. C.; Lin, L.; Chen, Y. T. Rapid Commun. Mass Spectrom. 1998, 12, 317-327.

(12) van Kampen, J. J.; Burgers, P. C.; de Groot, R.; Luider, T. M. Anal. Chem. 2006, 78, 5403-5411.

(13) van Kampen, J. J.; Verschuren, E. J.; Burgers, P. C.; Luider, T. M.; de Groot, R.; Osterhaus, A. D.; Gruters, R. A. I. Chromatogr. B: Anal. Technol. Biomed. Life Sci. 2007, 847, 38-44. 


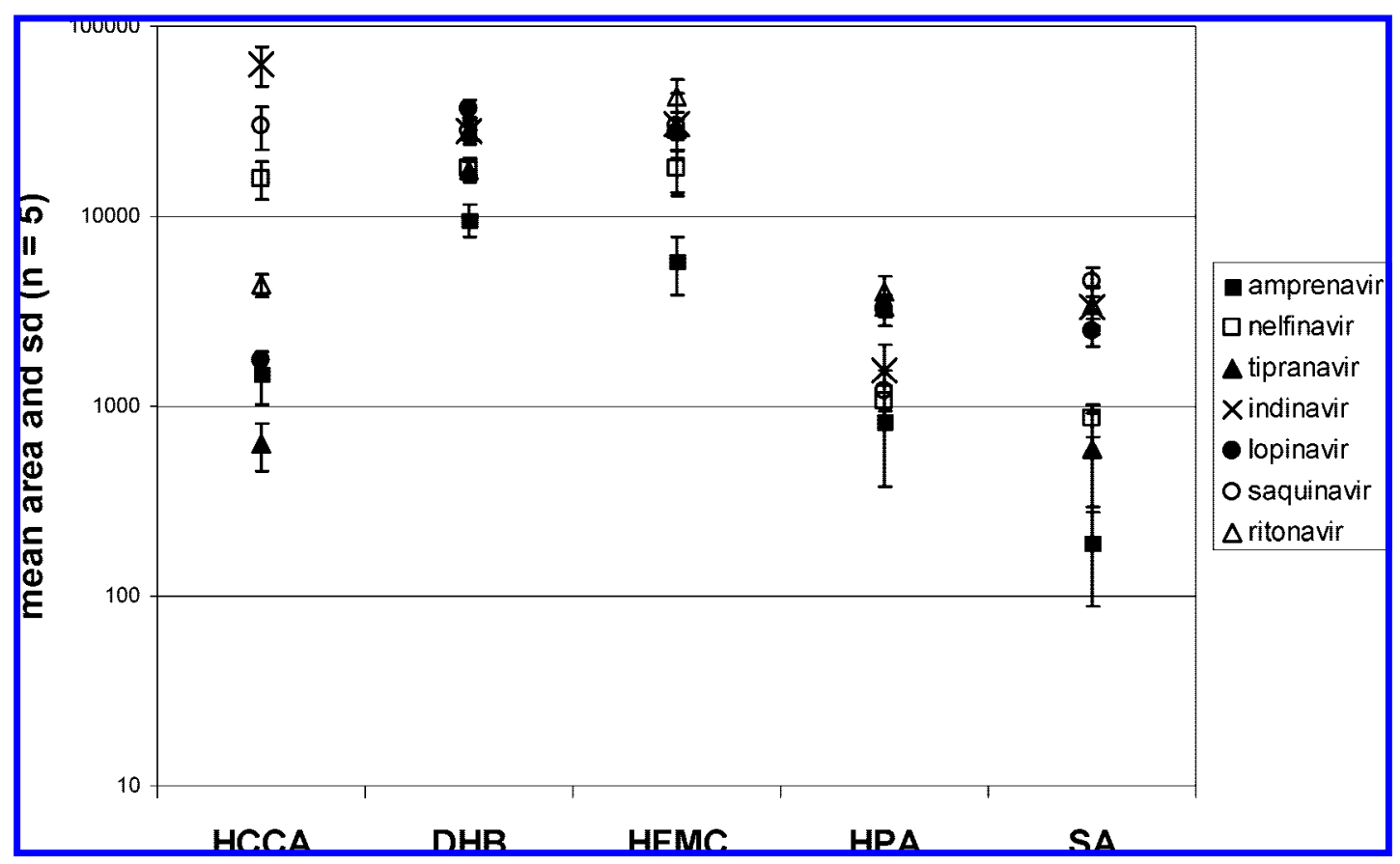

Figure 1. Comparison of signal intensities for HIV-1 protease inhibitors using various MALDI matrixes in combination with stainless steel target plates. Seven protease inhibitors were spiked in a lysate of $1 \times 10^{6}$ PBMC and analyzed using various matrixes. The amount of protease inhibitor per spot was 5 pmol. Reported are the mean areas and standard deviations of the SRM traces for a single spot (the areas for saquinavir were divided by 10).

target plates, ${ }^{14}$ fast evaporation protocols ${ }^{15}$ and sophisticated sample spotting devices. ${ }^{16}$ In addition, well-matched analytes and internal standards as well as a sufficient number of repeat measurements for signal averaging lead to significantly better precision values, resulting in the successful development of several quantitative drug assays using MALDI-TOF, ${ }^{12,13,17,18}$ MALDIqTOF ${ }^{19,20}$ MALDI-FTICR, and MALDI-triple quadrupole ${ }^{19,21-27}$ mass spectrometry.

In this study, we have explored the feasibility of using a prototype MALDI-triple quadrupole mass spectrometer for rapid quantitative analysis of HIV protease inhibitors and a non-

(14) Schuerenberg, M.; Luebbert, C.; Eickhoff, H.; Kalkum, M.; Lehrach, H.; Nordhoff, E. Anal. Chem. 2000, 72, 3436-3442.

(15) Vorm, O.; Roepstorff, P.; Mann, M. Anal. Chem. 1994, 66, 3281-3287.

(16) Hanton, S. D.; Hyder, I. Z.; Stets, J. R.; Owens, K. G.; Blair, W. R.; Guttman, C. M.; Giuseppetti, A. A. L. Am. Soc. Mass Spectrom. 2004, 15, 168-179.

(17) Duncan, M. W.; Matanovic, G.; Cerpa-Poljak, A. Rapid Commun. Mass Spectrom. 1993, 7, 1090-1094.

(18) Rideout, D.; Bustamante, A.; Siuzdak, G. Proc. Natl. Acad. Sci. U. S. A. 1993, 90, 10226-10229.

(19) Hatsis, P.; Brombacher, S.; Corr, J.; Kovarik, P.; Volmer, D. A. $\underline{\text { Rapid }}$ Commun. Mass Spectrom. 2003, 17, 2303-2309.

(20) Notari, S.; Mancone, C.; Tripodi, M.; Narciso, P.; Fasano, M.; Ascenzi, P. I. Chromatogr., B: Anal. Technol. Biomed. Life Sci. 2006, 833, 109-116.

(21) Corr, J. J.; Kovarik, P.; Schneider, B. B.; Hendrikse, J.; Loboda, A.; Covey, T. R. I. Am. Soc. Mass Spectrom. 2006, 17, 1129-1141.

(22) Gobey, J.; Cole, M.; Janiszewski, J.; Covey, T.; Chau, T.; Kovarik, P.; Corr, J. Anal. Chem. 2005, 77, 5643-5654.

(23) Kovarik, P.; Grivet, C.; Bourgogne, E.; Hopfgartner, G. Rapid Commun. Mass Spectrom. 2007, 21, 911-919.

(24) Signor, L.; Varesio, E.; Staack, R. F.; Starke, V.; Richter, W. F.; Hopfgartner, G. J. Mass Spectrom. 2007, 42, 900-909.

(25) Sleno, L.; Volmer, D. A. Rapid Commun. Mass Spectrom. 2006, 20, $1517-$ 1524.

(26) Sleno, L.; Volmer, D. A. Rapid Commun. Mass Spectrom. 2005, 19, 19281936.

(27) Sleno, L.; Volmer, D. A. Anal. Chem. 2005, 77, 1509-1517.

\section{Table 1. Quantitative Analysis of Antiretroviral Drugs in PBMC Lysate Using MALDI-QqQ ${ }^{a}$}

$\begin{array}{lccccc}\text { compound } & \begin{array}{c}\text { LLOQ } \\ \text { (pmol) }\end{array} & \begin{array}{c}\text { ULOQ } \\ \text { (pmol) }\end{array} & \begin{array}{c}\text { precision } \\ \text { (\% CV) }\end{array} & \begin{array}{c}\text { accuracy, RE } \\ \text { (\% deviation) }\end{array} & R^{2} \\ \text { nelfinavir } & 0.064 & 16 & 11.9(3.2) & 7.4(4.4) & 0.9998 \\ \text { indinavir } & 0.004 & 16 & 12.0(6.8) & 5.0(3.6) & 0.9999 \\ \text { saquinavir } & 0.016 & 4 & 10.8(3.2) & 4.3(3.4) & 0.9985 \\ \text { ritonavir } & 0.064 & 16 & 14.1(8.3) & 8.3(6.2) & 0.9975 \\ \text { lopinavir } & 0.064 & 16 & 12.9(4.6) & 6.7(4.4) & 0.9953 \\ \text { nevirapine } & 0.016 & 16 & 17.3(3.8) & 7.4(4.5) & 0.9999\end{array}$

${ }^{a}$ LLOQ, lower limit of quantitation in picomole per spot on the target plate. ULOQ, upper limit of quantification in picomole per spot on the target plate. Reported are the average precisions and accuracies (\% relative error, $\mathrm{RE}$ ) for data sets $\mathrm{A}$ and $\mathrm{B} . R^{2}=$ regression coefficient of the $1 / x$ weighed linear curve when data sets $A$ and $B$ were combined. Standard deviations are reported in parentheses. All experimental data in the table were obtained using DHB as maxtrix compound on an uncoated stainless steel target.

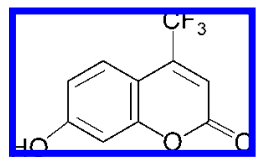

Figure 2. 7-Hydroxy-4(trifluoromethyl)coumarin (HFMC).

nucleoside reverse transcriptase inhibitor in lysates of peripheral blood mononuclear cells (PBMC).

\section{EXPERIMENTAL SECTION}

Chemicals. 7-Hydroxy-4-(trifluoromethyl)coumarin (HFMC), 2,5-dihydroxybenzoic acid (DHB), 3,5-dimethoxy-4-hydroxycinnamic acid (sinapinic acid; SA), 3-hydroxypicolinic acid (HPA), and $\alpha$-cyano-4-hydroxycinnamic acid (HCCA) were obtained from Sigma-Aldrich. The investigated drug compounds were kindly 


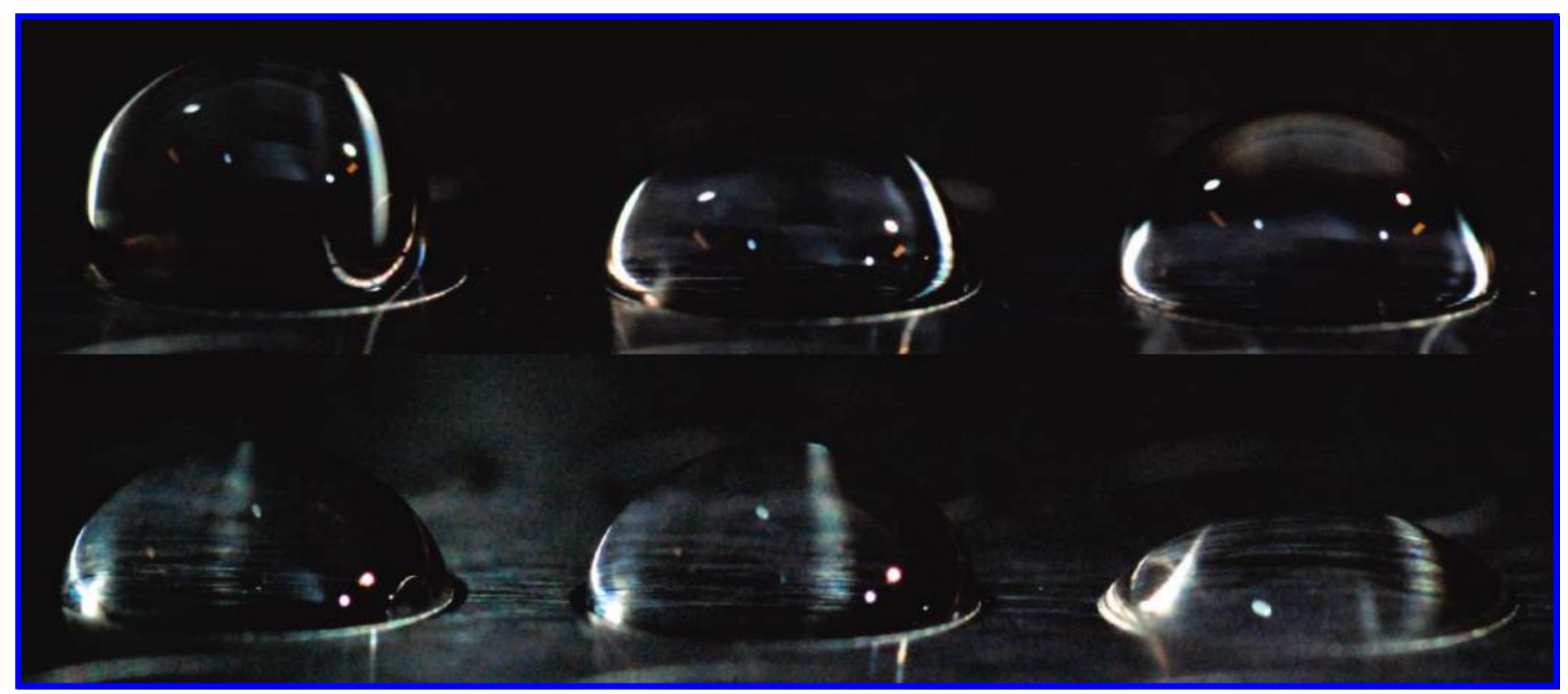

Figure 3. Droplets of $10 \mu \mathrm{L}$ of DHB solution in various solvents spotted on a fluoropolymer-coated MALDI target. Upper row: left, DHB in water; middle, DHB in ACN/water (1:1, v/v); right, $\mathrm{DHB}$ in $\mathrm{MeOH} /$ water (1:1, v/v). Lower row: left, $\mathrm{DHB}$ in $\mathrm{CAN}$; middle, DHB in MeOH; right, DHB in acetone.

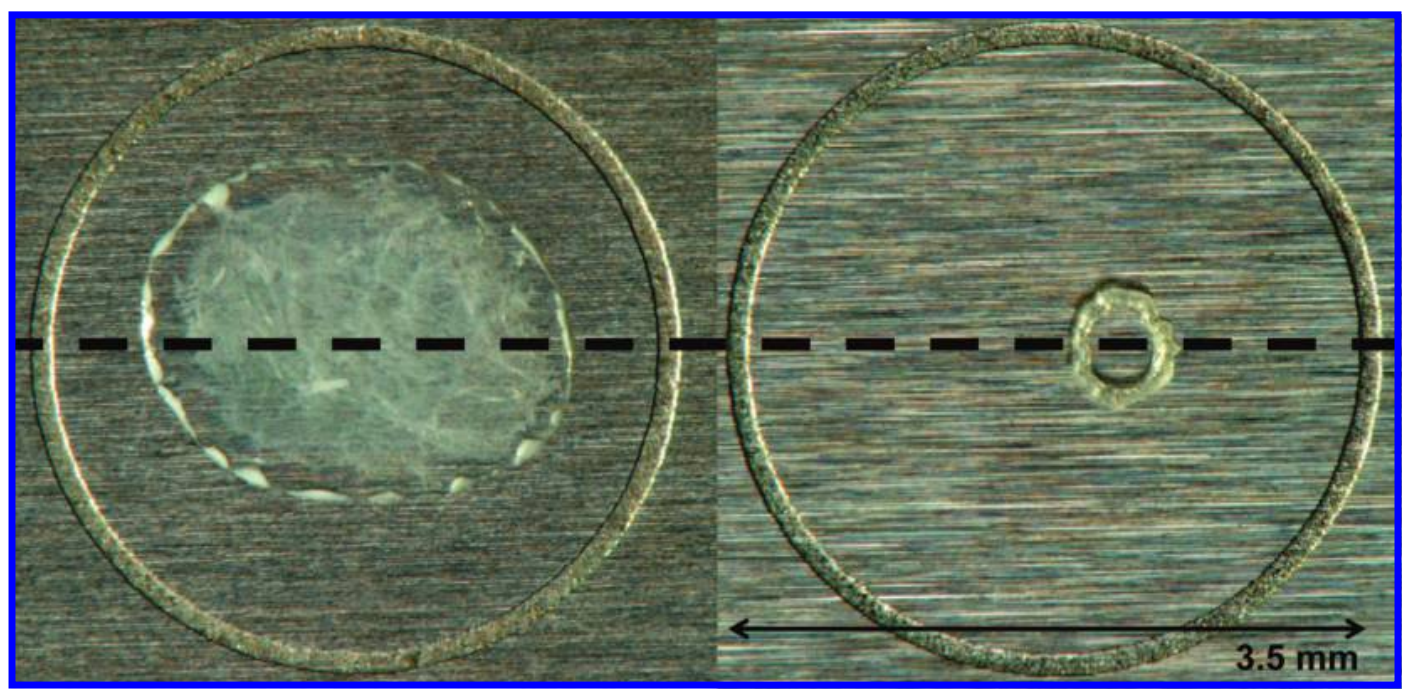

Figure 4. HFMC matrix spotted on to a stainless steel target plate and a fluoropolymer-coated target plate. Left: $1 \mu \mathrm{L}$ of HFMC matrix spotted on to a stainless steel target plate $\left(10 \mathrm{mg} / \mathrm{mL}\right.$ in $\left.\mathrm{ACN} / \mathrm{H}_{2} \mathrm{O}(1: 1, \mathrm{v} / \mathrm{v})\right)$. Right: $10 \mu \mathrm{L}$ of HFMC matrix spotted on to a fluoropolymer-coated target plate $(1 \mathrm{mg} / \mathrm{mL}$ in $100 \%$ acetonitrile). The dashed line represents the actual width of the laser beam (diameter, $\sim 110 \mu \mathrm{m})$. The etched circle is $\sim 3.5 \mathrm{~mm}$ wide.

donated by the following companies: lopinavir and ritonavir, Abbott Laboratories (Illinois, IL); saquinavir, F. Hoffmann-La Roche (Basel, Switzerland); nelfinavir, Pfizer (Groton, CT); indinavir Merck (Rahway, NJ); nevirapine and tipranavir, Boehringer Ingelheim (Ingelheim am Rhein, Germany); amprenavir, GlaxoSmithKline (Middlesex, United Kingdom). Carbamazepine was purchased from Sigma-Aldrich. The proprietary PFC1601V fluoropolymer solution was obtained from Cytonix Corp. (Beltsville, $\mathrm{MD})$. Eye protection and gloves should be worn when handling the fluoropolymer solution; there are no acute and chronic hazards to be expected for this product and no carcinogenic properties are known to be present (Material safety data sheet, Cytonix Corp.).

Mass Spectrometry. An API 3000 MALDI-triple quadrupole mass spectrometer (MDS Analytical Technologies, Concord, ON,
Canada) equipped with a prototype $1000-\mathrm{Hz} \mathrm{Nd}$ :YAG laser (355 nm) (PowerChip NanoLaser, JDS Uniphase, San José, CA) MALDI source was operated in the positive ion mode. The pressure in the MALDI source was $\sim 5$ mTorr. During laser firing, the target plate was moved horizontally at a constant speed $(\sim 0.06 \mathrm{~cm} / \mathrm{s})$ and the SRM traces of the analyte(s) and internal standard(s) were recorded (dwell time, $10 \mathrm{~ms}$ each). Analyst software version 1.1 (MDS Analytical Technologies) was used for data acquisition.

Sample Preparation. Peripheral blood mononuclear cells (PBMC) were isolated from a buffy coat (Sanquin, Rotterdam, The Netherlands) using a standard Ficoll density gradient. PBMC pellets were lysed in $100 \%$ methanol overnight at $5{ }^{\circ} \mathrm{C}(100 \mu \mathrm{L}$ of $\mathrm{MeOH}$ per $\left.1 \times 10^{6} \mathrm{PBMC}\right)$. The lysed $\mathrm{PBMC}$ were spun down at $14000 \mathrm{rpm}$ for $5 \mathrm{~min}$. Supernatants of $1 \times 10^{6} \mathrm{PBMC}$ were collected in $1.5-\mathrm{mL}$ vials, and $400 \mu \mathrm{L}$ of water was added to each 


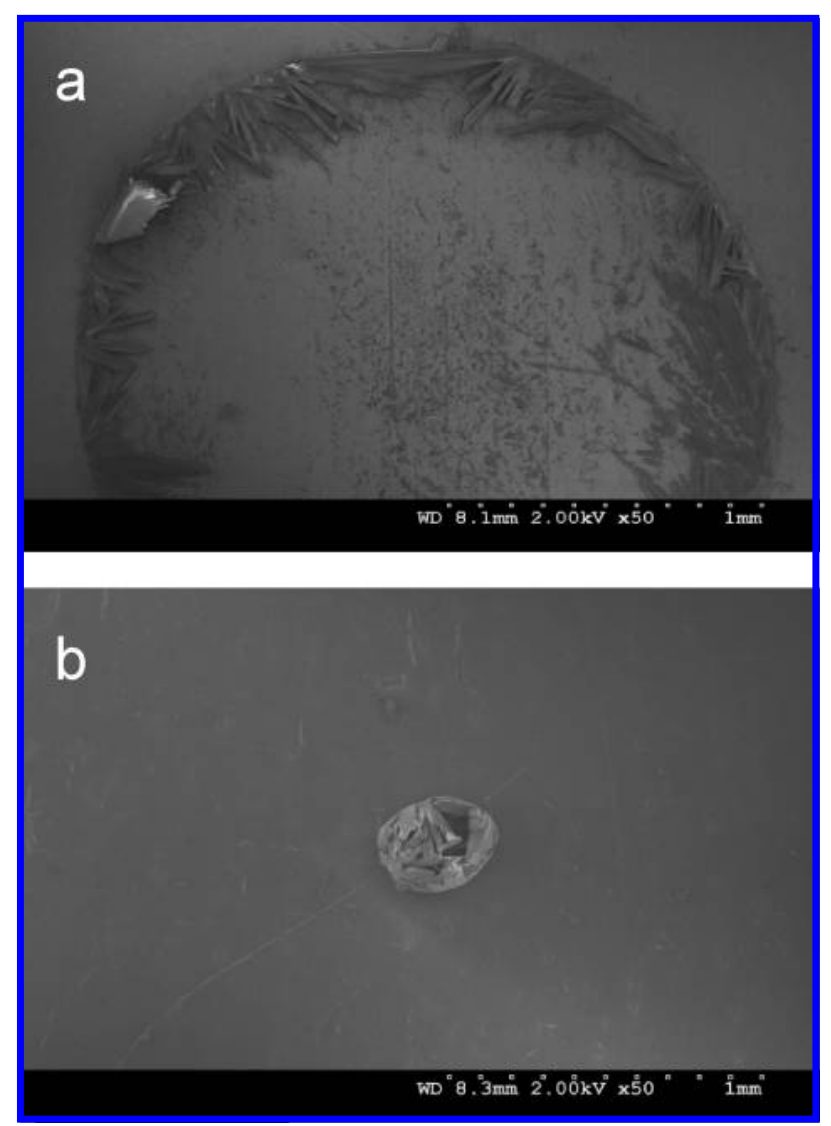

Figure 5. SEM analysis of dried sample spots. Spot size reduction for dispensed DHB solutions (25/75 acetonitrile/water): (a) $1 \mu \mathrm{L}$ on an uncoated stainless target; (b) $1 \mu \mathrm{L}$ on a coated plate. The diameter of the dried spot on the coated plate is $\sim 200 \mu \mathrm{m}$.

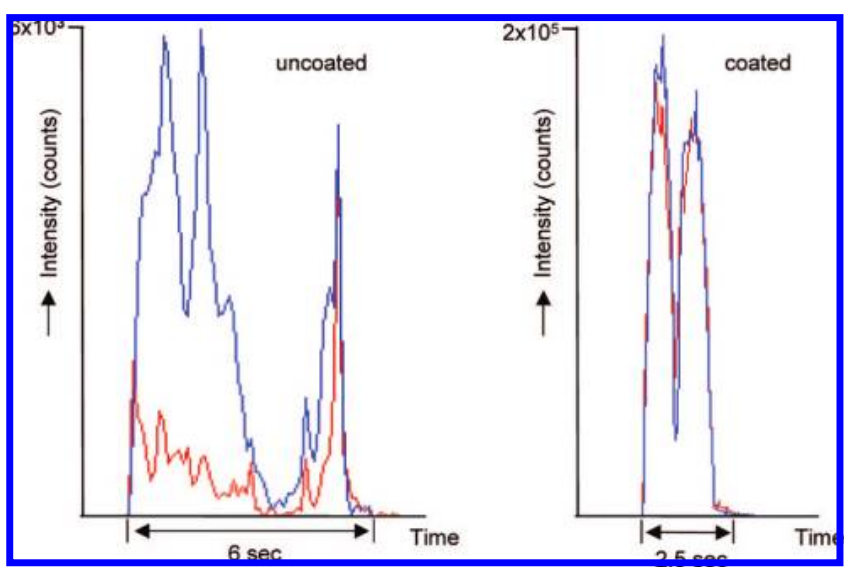

Figure 6. SRM traces of $4 \mathrm{pmol}$ of indinavir (blue) and $1 \mathrm{pmol}$ of saquinavir (red) in a single spot using the novel matrix HFMC on a stainless steel target plate (left) and on a fluoropolymer-coated target plate (right).

vial. Subsequently, the supernatants were cleaned using 96 -well solid-phase extraction (SPE) plates (Oasis HLB $\mu$ elution plate, Waters). Methanol and water ( $200 \mu \mathrm{L}$ each) were drawn through each well using a vacuum manifold according to the manufacturer's protocol. Subsequently, the samples were loaded onto the SPE plate and washed twice with methanol/water $(1: 4 \mathrm{v} / \mathrm{v})$. Finally, the samples were eluted from the plate with $100 \mu \mathrm{L}$ of methanol. Drug standards were added to the eluates and were subsequently dried using a SpeedVac (Savant).

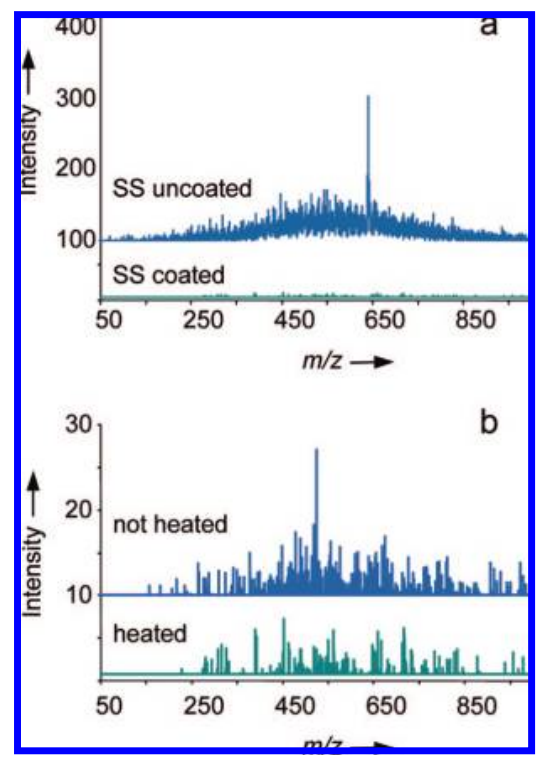

Figure 7. Comparison of background signals in the full-scan mode on uncoated and coated stainless steel (SS) plates (panel a). Panel $b$ shows the further improvements by heat curing the coated plates for $20 \mathrm{~min}$ at $200^{\circ} \mathrm{C}$.

On the day of analysis, the dried samples were reconstituted in $10 \mu \mathrm{L}$ of matrix solution and replicates of $1 \mu \mathrm{L}$ were spotted onto $10 \times 10$ stainless steel target plates (Perseptive Biosystems, Framingham, MA). For hydrophobically coated target plates, the dried samples were reconstituted in $100 \mu \mathrm{L}$ of matrix solution and replicates of $10 \mu \mathrm{L}$ were applied.

The following matrixes were used: $4 \mathrm{mg} / \mathrm{mL} \mathrm{HCCA}, 50 \mathrm{mg} /$ $\mathrm{mL}$ DHB, $10 \mathrm{mg} / \mathrm{mL}$ HFMC, $50 \mathrm{mg} / \mathrm{mL} \mathrm{HPA}$, and $4 \mathrm{mg} / \mathrm{mL} \mathrm{SA}$. All matrixes were dissolved in acetonitrile/water/formic acid (500: $500: 1 ; \mathrm{v} / \mathrm{v} / \mathrm{v})$. For the HFMC matrix in combination with the hydrophobic target plate, $1 \mathrm{mg} / \mathrm{mL}$ HFMC in acetonitrile/formic acid (200:1; v/v) was used.

Scanning Electron Microscope (SEM). SEM photos were obtained on a Hitachi S-3000N SEM (Hitachi Science Systems, Hitachinaka, Japan). Various levels of magnification were used at $2.00-\mathrm{kV}$ electron energy. Spot areas were determined using SimplePCI software (version 4.0.0, Compix Inc. Imaging Systems, Cranberry Township, PA).

Preparation of Target Plate with Hydrophobic Coating. The target plates were spin-coated with a hydrophobic coating using a modified centrifuge with a small hole in the top lid and a custom-fitted Teflon rotor for the MALDI plates. During spinning of the target plate at $1000 \mathrm{rpm}, 500 \mu \mathrm{L}$ of PFC $1601 \mathrm{~V}$ coating solution was slowly applied through the hole to the center of the target plate. Subsequently, the target plate was spun for $1 \mathrm{~min}$ to evenly distribute the coating solution. The coated plate was then heated for $20 \mathrm{~min}$ at $\sim 200^{\circ} \mathrm{C}$. The coating material can also be applied onto the target plate by simply using a brush, with a slight compromise in coating homogeneity.

Quantitative Analysis. The following transitions were monitored for SRM: lopinavir, $630.5 \rightarrow 183$ (collision energy, $30 \mathrm{eV}$ ); ritonavir, $722 \rightarrow 296(30 \mathrm{eV})$; saquinavir, $671.5 \rightarrow 570.5(40 \mathrm{eV})$; amprenavir, $506.5 \rightarrow 245.5(30 \mathrm{eV})$; indinavir, $614.5 \rightarrow 421.5(40$ $\mathrm{eV})$; nelfinavir, $569 \rightarrow 331(40 \mathrm{eV})$; tipranavir, $603.5 \rightarrow 411(30$ $\mathrm{eV})$; nevirapine, $267 \rightarrow 226(30 \mathrm{eV})$; carbamazepine, $237 \rightarrow 194$ $(30 \mathrm{eV})$. As internal standard, carbamazepine for nevirapine, 


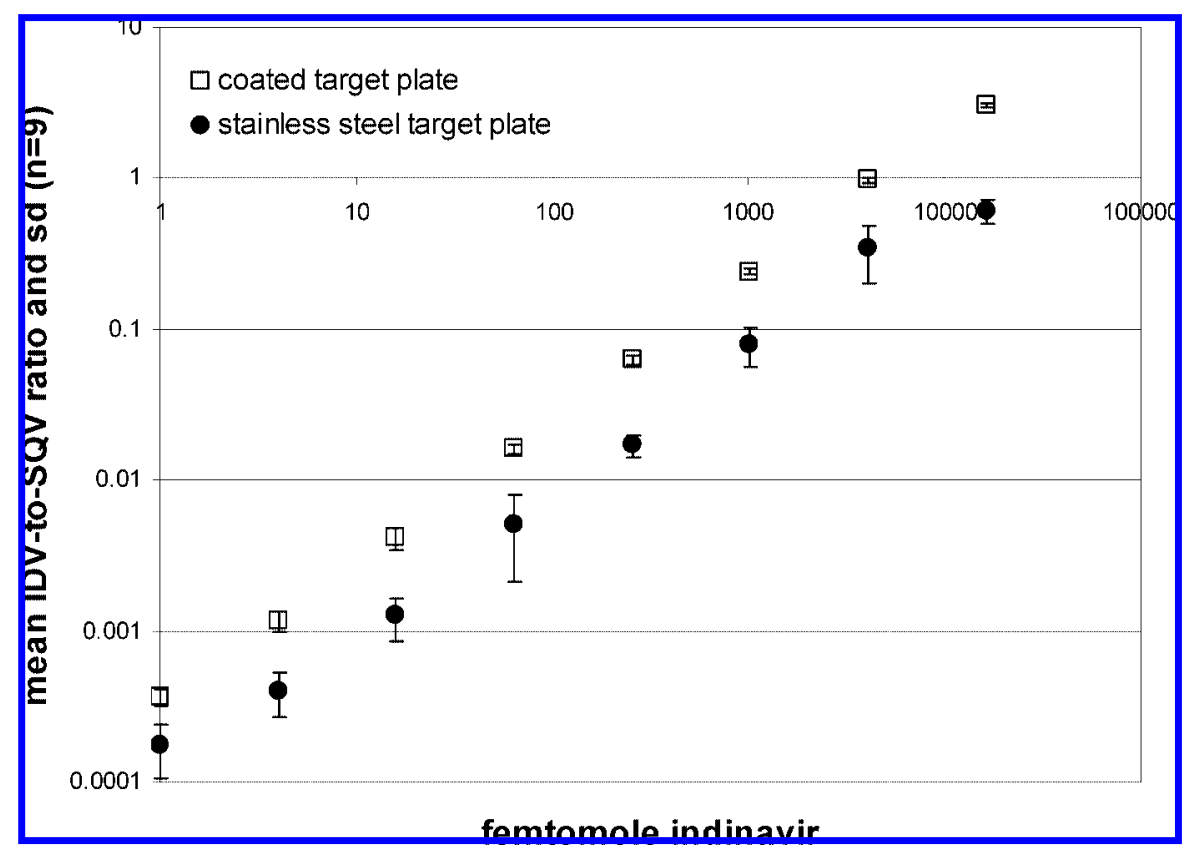

Figure 8. Quantitative analysis of indinavir in PBMC lysates using the novel matrix HFMC on a stainless steel target plate and on a fluoropolymercoated plate.

indinavir for amprenavir, lopinavir for tipranavir, indinavir for nelfinavir, saquinavir for ritonavir, nelfinavir for indinavir, and tipranavir for lopinavir were used.

Two data sets, $\mathrm{A}$ and $\mathrm{B}$, were measured, each containing five replicate spots per analyte. Data set A was used to construct the calibration curve while data set B served as quality control, and vice versa. For each spot, the peak area ratios of analyte to internal standard were measured. Subsequently, the average analyte/ internal standard ratios for the replicate analyses were calculated, and used for the calibration curves and for quality control purposes. Linear $1 / x$-weighed curves were used for calibration, unless otherwise stated.

\section{RESULTS AND DISCUSSION}

In MALDI experiments, the nature of the matrix compound has a crucial influence on the ionization efficiencies and the signal intensities of the analytes of interest. ${ }^{28}$ For example, DHB and HCCA are commonly used for analysis of peptides and small proteins, while SA is preferred for larger proteins. When a small molecule is used as matrix, many of the abundant peaks observed in the low-mass range of the MALDI mass spectrum arise from the matrix itself. Upon laser irradiation, matrix molecules undergo a variety of different processes such as ionization and subsequent fragmentation, photodissociation, and ion-molecule reactions, leading to matrix-derived chemical noise in the low- $m / z$ range. These interfering signals seriously hamper the analysis of small molecules with $m / z$ values below 1000 . Various approaches have been described to overcome this limitation, for example, by carefully choosing the matrix-to-analyte ratio, ${ }^{4}$ the use of additives, ${ }^{5}$ ionic liquids, ${ }^{6}$ or inorganic matrixes. ${ }^{7,8}$ The use of high molecular weight porphyrin matrixes can also decrease the matrixderived chemical noise in the low- $m / z$ range. ${ }^{10,11,29}$ We have previously shown that using the high molecular weight porphyrin

(28) Gross, J. H. In Mass Spectrometry: A Textbook, 1st ed.; Gross, J. H., Ed.; Springer: New York, 2004. matrix meso-tetrakis(pentafluorophenyl)porphyrin (F20TPP) results in a significant decrease of the matrix-related peaks in the low- $m / z$ range in MALDI-TOF analyses and that this matrix can be used for quantitative analysis of HIV-1 protease inhibitors in cell lysates. ${ }^{12,13}$ By contrast, in MALDI-FTICR mass spectrometry, the high molecular weight matrix F20TPP undergoes extensive dissociation on the time scale of the FTICR experiment $(\sim 1 \mathrm{~s})$ and intense fragment ions are observed in the low mass range, which leads to interfering chemical noise. Significantly less fragmentation of such ions is observed in MALDI-TOF mass spectrometers $\left(10^{-4} \mathrm{~s}\right)$. Thus, for the quantitative analysis of small molecules, a high molecular weight matrix was used for TOF analyses and a low molecular weight matrix for the FTICR experiments, showing that the type of mass analyzer can have a significant influence on the choice of matrix for small-molecule analysis by MALDI. Another efficient way for eliminating the chemical noise is the application of MS/MS, by monitoring only analyte-specific ion transitions. This technique is particularly useful when a triple quadrupole MS is used for MS/MS. In the SRM mode of the QqQ instrument, a nearly 100\% duty cycle is obtained, and this makes it extremely efficient for small molecule target analysis. Using this technique, a wide variety of matrixes, such as HCCA and DHB, have been used for small-molecule analysis. Sleno and Volmer compared HCCA, DHB, and SA for analysis of spirolide toxins, quinidine, danofloxacin, ramipril, and nadolol and found that HCCA yielded the strongest signal intensities and the best precision for these drugs. ${ }^{26,27}$ In addition, Gobey et al. showed that HCCA resulted in successful detection of 175 out of the 208 tested compounds. ${ }^{22}$

Comparison of Matrixes. In the present study, we have compared the use of the HCCA, DHB, SA, 3-hydroxypicolinic acid (HPA), and a new matrix, 7-hydroxy-4-(trifluoromethyl)coumarin (HFMC), for the analysis of seven HIV-1 protease inhibitors by

(29) Ayorinde, F. O.; Hambright, P.; Porter, T. N.; Keith, Q. L., Jr. Rapid Commun. Mass Spectrom. 1999, 13, 2474-2479. 
MALDI-QqQ (Figure 1). SA and HPA yielded very poor signal intensities in comparison to HCCA, DHB, and HFMC. HCCA exhibited intense signals only for nelfinavir, indinavir, and saquinavir, but relatively weak signals for lopinavir, ritonavir, amprenavir, and tipranavir. DHB and the new matrix HFMC on the other hand generated very strong signals for all seven protease inhibitors tested. Using MALDI-TOF and MALDI-FTICR, we have previously shown that abundant signals for antiretroviral drugs can be obtained by using a cationization approach with alkali halides. ${ }^{12,13}$ Using MS/MS on the MALDI-QqQ instrument, however, we found that the cationized drugs showed poor fragmentation efficiencies and thus low SRM sensitivities compared to the protonated precursor molecules. In general, we found that higher collision energies were needed for fragmentation of the cationized drugs as compared to the protonated forms.

Quantitative Analysis of Antiretroviral Drugs in Cell Lysates using DHB Matrix. We explored DHB as matrix for quantitative analysis of the protonated forms of the HIV protease inhibitors nelfinavir, saquinavir, indinavir, lopinavir, and ritonavir and the non-nucleoside reverse transcriptase inhibitor nevirapine in lysates of PBMC (Table 1). The measurement precisions were relatively high compared to LC-MS/MS methods for antiretroviral drugs in PBMC lysates. ${ }^{30}$ Sleno and Volmer showed that using HCCA resulted in significantly better precisions as compared to DHB, because of the more homogeneous crystal layers formed from HCCA solutions. ${ }^{26,27}$ In our experiments, however, the signal intensities for antiretroviral drugs were much better for DHB as matrix as compared to HCCA (vide supra), necessitating DHB as matrix. Unfortunately, no stable isotope standards were commercially available for the analytes investigated here, to compensate for the heterogeneous crystallization. Alternatively, we used chemically similar protease inhibitors as internal standard for analysis of the protease inhibitors of interest (see Experimental Section) and carbamazepine as a chemically related internal standard for the non-nucleoside reverse transcriptase inhibitor nevirapine. Applying stable isotope standards generally results in significantly better precisions, since the chemical properties of internal standard and analyte are virtually identical. This is particularly important for MALDI, as well-matched analyte and internal standard pairs result in homogeneous cocrystallizations, as recently shown by Sleno and Volmer. ${ }^{25}$ It was also shown in that study, however, that structural analogues of the analyte could be successfully used, as long as important solution-phase properties were appropriately matched. To compensate for the relatively high fluctuations mentioned above, we measured and averaged five replicates of each sample and used the mean analyte-tointernal standard ratio to calculate the concentration in the sample. The resulting average relative errors ranged between $5.0 \%$ for indinavir to $8.3 \%$ for ritonavir. The lower limits of quantitation, expressed as amount of drug per spot on the target plate, was 64 fmol for nelfinavir, $4 \mathrm{fmol}$ for indinavir, $16 \mathrm{fmol}$ for saquinavir, 64 fmol for lopinavir, $64 \mathrm{fmol}$ for ritonavir, and $16 \mathrm{fmol}$ for nevirapine. These numbers readily allow the clinical application of the technique.

Coumarin Matrix and Fluoropolymer-Coated Target Plates. We also explored the use of the new matrix compound HFMC

(30) Colombo, S.; Beguin, A.; Telenti, A.; Biollaz, J.; Buclin, T.; Rochat, B.; Decosterd, L. A. I. Chromatogr. B: Anal. Technol. Biomed. Life Sci. 2005, 819, 259-276.
(Figure 2) for quantitative analysis of indinavir in PBMC lysates. HFMC is a photoluminescent molecule with a molecular mass of $230.02 \mathrm{Da}$. HFMC exhibits maximum light absorption at $355 \mathrm{~nm}$, which ideally matches the wavelength of the frequency-tripled, solid-state Nd:YAG laser of the MALDI-QqQ system $(\lambda=355 \mathrm{~nm})$. Upon excitation, HFMC emits fluorescent light at $\lambda=498 \mathrm{~nm}$. In acidic solutions, HFMC is colorless, while it is bright yellow in basic solutions. When the HFMC matrix was spotted on a stainless steel target plate using the dried droplet technique, the quantitative precisions and quantitative accuracies were poor. Inspection of the SRM traces showed differential incorporation of analyte and internal standard in the HFMC crystals. A fast evaporation approach, ${ }^{15}$ i.e., spotting from $100 \%$ organic solvents, was used to obtain a more homogeneous distribution of analyte and internal standard in the HFMC crystals. However, extensive spreading of the sample was observed when the fast evaporation protocol was applied in combination with stainless steel target plates. It has been previously shown that hydrophobic coatings can significantly improve crystallization behavior on MALDI plates. ${ }^{31-35}$ In this study, we coated the target plate with an inexpensive strongly hydrophobic fluoropolymer ( $\sim$ US $\$ 0.20 /$ plate). The coated target plates allowed spotting of large volumes of sample, even for $100 \%$ organic solutions of up to $10 \mu \mathrm{L}$ (Figure 3). Furthermore, even these large volumes of sample dried to very small crystal areas on the target plates, due to the remarkable focusing abilities of the coating material (Figure 4). The reduction factor in the area of the crystal layers was further investigated by spotting droplets of $1-\mu \mathrm{L}$ DHB solutions in acetonitrile/water mixtures of varying composition on coated as well as uncoated target plates (Figure 5). Using the coated target plates, the area of the dried spots reduced by a factor 18.2 (acetonitrile content of 75\%), 23.3 (50\% $\mathrm{ACN})$, and $31.5(25 \% \mathrm{ACN})$, which is significantly larger than previously reported coating materials. ${ }^{31-35}$ Figure 6 illustrates that the coated target plates significantly improve the cocrystallization of analyte and internal standard for indinavir when HFMC is used as matrix. In addition, the measurement times for one spot were reduced from 6 to $2.5 \mathrm{~s}$. Another feature of the coating is a significant reduction in background signals from contaminants on the target plate (Figure 7). Figure 8 illustrates the improvement in the concentration-response curve for indinavir in PBMC lysates as well as improvement in precision when the fluoropolymer-coated plate was used in combination with the HFMC matrix. Using the fluoropolymer-coated plate, the average precision improved from 34 (sd 17.6) to $9.9 \% \mathrm{CV}$ (sd 5.7) and the relative error went down from 10.1 (sd 4.8) to 4.6\% (sd 3.6). In addition, the lower limit of quantitation improved from 16 to $1 \mathrm{fmol}$ per spot on the target plate using a $1 / x^{2}$-weighed linear curve.

\section{CONCLUSIONS}

There is an increasing interest in the use of MALDI mass spectrometry for quantitative analysis of small molecules, due to

(31) Owen, S. J.; Meier, F. S.; Brombacher, S.; Volmer, D. A. Rapid Commun. Mass Spectrom. 2003, 17, 2439-2449.

(32) Gundry, R. L.; Edward, R.; Kole, T. P.; Sutton, C.; Cotter, R. J. Anal. Chem. 2005, 77, 6609-6617.

(33) Konig, S.; Grote, J. Biotechniques 2002, 32, 912914-915.

(34) Redeby, T.; Roeraade, J.; Emmer, A. Rapid Commun. Mass Spectrom. 2004, 18, 1161-1166.

(35) Xiong, S.; Ding, Q.; Zhao, Z.; Chen, W.; Wang, G.; Liu, S. Proteomics 2003, 3, 265-272. 
its very high sample throughput capability, its insensitivity to ion suppression, and the possibility of storing samples on target plates for future use or reanalysis. Using a prototype MALDI-QqQ mass spectrometer, we have shown here that this technique can be used for accurate quantitative analysis of HIV protease inhibitors and a non-nucleoside reverse transcriptase inhibitor in lysates of 1 million peripheral blood mononuclear cells. The limits of quantitation readily allows clinical applications of the technique. In this study, we had to resort to chemical analogues as internal standards, because stable isotope internal standards were not available. Stable isotopes are often very expensive and are frequently not commercially available, and the custom synthesis of these compounds is laborious. While using chemical analogues as internal standards may not at first appear to be the best choice, it is frequently the only practical option for quantitative analysis using LC-MS or MALDI-MS. In our study, we demonstrated that chemically similar compounds as standards and replicate analyses yielded excellent precision and accuracy. We have also shown that the optimum matrix for specific analytes can be highly compound dependent. By using a novel matrix and a fluoropolymer surface coating, we were able to achieve improved cocrys- tallization of analyte and internal standard, thus further improving precisions and accuracies.

\section{ACKNOWLEDGMENT}

Financial support was obtained from Aids Fund, The Netherlands (project 2004051 (J.J.A.v.K.), and the VIRGO consortium, an Innovative Cluster approved by The Netherlands Genomics Initiative and partially funded by the Dutch Government (BSIK 03012). Top Institute Pharma (project T4-212) and the members of project T4-212 (Erasmus MC (Rotterdam), TNO Quality of Life (Zeist), UMC St. Radboud (Nijmegen), and GlaxoSmithKline (Zeist)) are acknowledged for their scientific support. D.A.V. appreciates research support to his group from MDS Analytical Technologies, including the prototype MALDI triple quadrupole instrument. Frank van der Panne (Pathology, Erasmus MC) and Jan Tuin (Thorax Center, Erasmus MC) are gratefully acknowledged for their photography skills and Nicole Zehethofer, Andrew Leslie, Rambod Daneshfar and Felix Meier for their technical support. T.M.L. and D.A.V. contributed equally to this work.

Received for review January 30, 2008. Accepted May 8, 2008.

AC800218A 